\title{
Frontal and occipital horn ratio is associated with multifocal intraparenchymal hemorrhages in neonatal shunted hydrocephalus
}

\author{
*Soliman Oushy, BS, ${ }^{1}$ Jonathon J. Parker, MD, PhD, ${ }^{2}$ Kristen Campbell, MS, ${ }^{6,8}$ \\ Claire Palmer, MS, ${ }^{7,8}$ Corbett Wilkinson, MD, ${ }^{1,3}$ Nicholas V. Stence, MD, ${ }^{4,5}$ \\ Michael H. Handler, MD, ${ }^{1,3}$ and David M. Mirsky, MD ${ }^{4,5}$
}

\begin{abstract}
Departments of ${ }^{1}$ Neurosurgery, ${ }^{4}$ Radiology, and ${ }^{7}$ Pediatrics, University of Colorado School of Medicine; Departments of ${ }^{3}$ Neurosurgery and ${ }^{5}$ Radiology and ${ }^{8} \mathrm{Child}$ Health Research Biostatistical Core, Children's Hospital Colorado; and ${ }^{6}$ Department of Biostatistics and Informatics, Colorado School of Public Health, Aurora, Colorado; and 'Department of Neurosurgery, Stanford University, Stanford, California
\end{abstract}

\begin{abstract}
OBJECTIVE Placement of a cerebrospinal fluid diversion device (i.e., shunt) is a routine pediatric neurosurgical procedure, often performed in the first weeks of life for treatment of congenital hydrocephalus. In the postoperative period, shunt placement may be complicated by subdural, catheter tract, parenchymal, and intraventricular hemorrhages. The authors observed a subset of infants and neonates who developed multifocal intraparenchymal hemorrhages (MIPH) following shunt placement and sought to determine any predisposing perioperative variables.
\end{abstract}

METHODS A retrospective review of the electronic medical record at a tertiary-care children's hospital was performed for the period 1998-2015. Inclusion criteria consisted of shunt placement, age < 30 days, and available pre- and postoperative brain imaging. The following data were collected and analyzed for each case: ventricular size ratios, laboratory values, clinical presentation, shunt and valve type, and operative timing and approach.

RESULTS A total of 121 neonates met the inclusion criteria for the study, and 11 patients (9.1\%) had MIPH following shunt placement. The preoperative frontal and occipital horn ratio (FOR) was significantly higher in the patients with MIPH than in those without ( 0.65 vs $0.57, p<0.001)$. The change in FOR $(\Delta F O R)$ after shunt placement was significantly greater in the MIPH group (0.14 vs $0.08, p=0.04$ ). Among neonates who developed MIPH, aqueductal stenosis was the most common etiology (45\%). The type of shunt valve was associated with incidence of MIPH $(p<0.001)$. Preoperative clinical parameters, including head circumference, bulging fontanelle, and coagulopathy, were not significantly associated with development of MIPH.

CONCLUSIONS MIPH represents an underrecognized complication of neonatal shunted hydrocephalus. Markers of severity of ventriculomegaly (FOR) and ventricular response to CSF diversion ( $\triangle F O R$ ) were significantly associated with occurrence of MIPH. Choice of shunt and etiology of hydrocephalus were also significantly associated with MIPH. After adjusting for corrected age, etiology of hydrocephalus, and shunt setting, the authors found that $\triangle F O R$ after shunting was still associated with MIPH. A prospective study of MIPH prevention strategies and assessment of possible implications for patient outcomes is needed.

https://thejns.org/doi/abs/10.3171/2017.6.PEDS16481

KEY WORDS hydrocephalus; shunt; hemorrhage; neonatal

$\mathrm{I}$ MPLANTATION of a cerebrospinal fluid (CSF) diversion device, such as a shunt or external ventricular drain (EVD), is one of the most common procedures performed in pediatric neurosurgery. ${ }^{1,15}$ Shunting is frequently used in the treatment of hydrocephalus of various etiologies, including congenital hydrocephalus. Immediate and delayed complications of CSF diversion have been well studied and reviewed elsewhere. Complications include shunt malfunction, infection, intraparenchymal hemorrhage, intraventricular hemorrhage, and subdural collections., ${ }^{9,16,17}$

ABBREVIATIONS CM-II = Chiari Type II malformation; CSF = cerebrospinal fluid; ETV = endoscopic third ventriculostomy; EVD = external ventricular drain; FOR = frontal and occipital horn ratio; INR = international normalized ratio; IPH = intraparenchymal hemorrhage(s); MIPH = multifocal IPH; US = ultrasonography; VP = ventriculoperitoneal; $\triangle \mathrm{FOR}=$ change in FOR.

SUBMITTED August 22, 2016. ACCEPTED June 14, 2017.

INCLUDE WHEN CITING Published online September 8, 2017; DOI: 10.3171/2017.6.PEDS16481.

* Mr. Oushy and Dr. Parker share first authorship. 
The development of multifocal intraparenchymal hemorrhages (MIPH) after shunt placement is infrequently reported, and the etiology is not well understood. Unlike intraventricular and catheter-related hemorrhages, MIPH can occur in a delayed fashion outside of the immediate postoperative period. In previous reports, MIPH has been observed more often in infants younger than 3 months with severe hydrocephalus and no prior history of intracranial surgery. ${ }^{2}$ The hemorrhages are observed at the gray and white matter junction and in the periventricular region throughout the cerebral hemispheres (Fig. 1). ${ }^{2}$ Both arteriolar and venous etiologies for these hemorrhages have been hypothesized. ${ }^{2,12,18}$ The risk factors for and clinical significance of these hemorrhages are unknown. The authors sought to characterize patients who developed MIPH following CSF diversion and to determine any predisposing perioperative variables to guide future practices for prevention of this complication.

\section{Methods}

A retrospective review of the electronic medical record at a tertiary-care children's hospital was performed for the period 1998-2015. Inclusion criteria consisted of the following: shunt or EVD placement or endoscopic third ventriculostomy (ETV), age < 30 days at time of surgery, and available pre- and postoperative brain imaging. Patients with prior shunt placement and hydranencephaly were excluded from the study. For each patient, the following information was collected and analyzed: ventricular size ratios, laboratory values (platelet count and international normalized ratio [INR]), clinical presentation (head circumference and anterior fontanelle fullness), type of CSF diversion (ventriculoperitoneal [VP] shunt, ventriculosubgaleal shunt, EVD, or endoscopic third ventriculostomy [ETV]), and valve type as well as operative timing and approach. Corrected ages were calculated by adding the gestational age to the chronological age at the time of surgery. This study was approved by the Colorado Multiple Institutional Review Board (COMIRB) to ensure protection of human research subjects.

\section{Shunt Valves}

For all patients included in the study, information was available regarding the manufacturer and type of valve used in addition to the shunt setting, if applicable. Our classification system reflects the 2 leading mechanisms of implanted shunt valves-pressure control or flow control. The pressure control valves were substratified into low, medium, and high opening pressures (Table 1).

\section{Imaging}

Perioperative imaging modalities included MRI, CT, and ultrasonography (US). All patients had brain imaging available within 2 weeks following their CSF diversion procedure. Patients with a suspicious finding on US had subsequent CT or MRI to exclude acute hemorrhage. Preoperative imaging was used to determine the etiology of hydrocephalus. The different etiologies were classified as Chiari Type II malformation (CM-II)/myelomeningocele, intraventricular hemorrhage, aqueductal stenosis, arach-

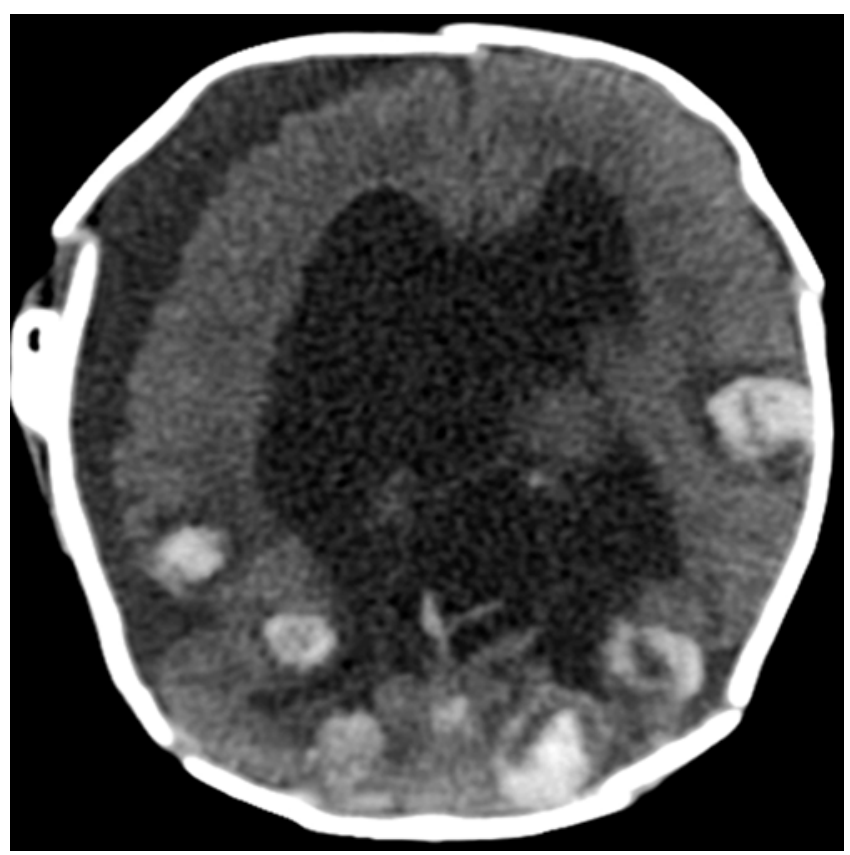

FIG. 1. Axial CT image demonstrating MIPH at the gray and white matter junction following shunt placement.

noid and ventricular malformations (e.g., cysts), DandyWalker malformation, tumor, or other (includes unknown etiology). Chiari Type II malformations were grouped with myelomeningocele, given their near $100 \%$ association.

The frontal and occipital horn ratio (FOR) is a measure of hydrocephalus severity that has been validated across multiple imaging modalities..$^{10}$ This ratio is defined in part by the width of the frontal and occipital horns of the lateral ventricle, and it is particularly accurate for capturing the severity of hydrocephalus across ventricular phenotypes, especially in colpocephalic patients, with disproportionate enlargement of the occipital horns of the lateral ventricles. Available perioperative MRI, CT, and US images were used to measure the maximum width of the biparietal diameter and maximum width of the occipital and frontal horns of the lateral ventricles (Fig. 2). The FOR was obtained by dividing the sum of the measured width of the frontal and occipital lateral horns by the measured biparietal diameter of the brain on pre- and postoperative images. The FOR measurement technique was performed on 10 sample postoperative images by 2 board-certified pediatric neuroradiologists (D.M.M., N.V.S.) with a high degree of interrater reliability. The single-measure intraclass correlation was 0.959 (95\% CI 0.845-0.990, p < 0.001). For the minority of cases with uncertainty in regard to etiology of hydrocephalus, the medical records were reviewed by neuroradiologist and neurosurgeon authors to achieve consensus. The designation of MIPH was restricted to newly developed postoperative hemorrhages of 3 or more foci, distant from the ventricular catheter and tract, as well as unrelated to an underlying vascular malformation. All imaging studies were reviewed independently by 2 pediatric neuroradiologists (N.V.S. and D.M.M.). 
TABLE 1. Shunt valve classification used in our cohort

\begin{tabular}{|c|c|c|c|}
\hline Low-Pressure Valves & Medium-Pressure Valves & High-Pressure Valves & Flow-Control Valves \\
\hline $\begin{array}{l}\text { Codman Certas Plus valve (performance } \\
\text { setting: 1) }\end{array}$ & $\begin{array}{l}\text { Codman Certas Plus valve (perfor- } \\
\text { mance setting: } 2-3 \text { ) }\end{array}$ & $\begin{array}{l}\text { Codman Certas Plus valve } \\
\text { (performance setting: } 4-6 \text { ) }\end{array}$ & $\begin{array}{l}\text { Integra OSV II valve system } \\
\text { Integra OSV II Low Pro }\end{array}$ \\
\hline Codman Hakim Valve $(40$ mm Hg) & Integra Equi-Flow valve (performance & Medtronic Strata (opening & valve system \\
\hline Integra Novus (low pressure) & level 1.5) & pressure: 2.5$)$ & \\
\hline Medtronic Delta (performance levels: 0.5-1.0) & Integra Novus (medium pressure) & & \\
\hline Medtronic Strata (opening pressures: $0.5-1.0$ ) & $\begin{array}{l}\text { Medtronic Strata (opening pressures: } \\
1.5-2.0 \text { ) }\end{array}$ & & \\
\hline
\end{tabular}

\section{Statistical Methods}

Demographic and clinical characteristics were summarized using counts and proportions for categorical variables and means and standard deviations for continuous variables. Two-sample independent t-tests and Fisher exact tests were used to test for differences between the group of patients who developed MIPH and the group of those who did not, for continuous and categorical clinical variables, respectively. ANOVA was used to test the association between FOR measurements and shunt classification and etiology of hydrocephalus. Tukey's honestly significant difference was used for post hoc testing. Two multivariable logistic regression models were fit, one with preoperative FOR as the primary explanatory variable and one with $\triangle F O R$ as the primary explanatory variable. Both had MIPH as the outcome and were adjusted for hydrocephalus etiology (with the combined categories of CMII, aqueductal stenosis, and all others), shunt setting, and corrected age. All hypothesis tests were 2-sided with significance set at 0.05. $\mathrm{R}$ (The $\mathrm{R}$ Foundation) version 3.3.1 software was used for statistical analysis.

\section{Results \\ Study Population}

A total of 158 neonates underwent CSF diversion procedures between 1998 and 2015; 37 neonates were excluded based on defined study criteria. A total of 121 patients met the inclusion criteria, and 11 of these patients $(9.1 \%)$ were diagnosed with MIPH. A comparison of patient characteristics between the MIPH group and the 110 patients without MIPH (control group) is shown in Table 2. There were no significant differences between the 2 groups in terms of sex, corrected age, prematurity, head circumference, anterior fontanelle, or gestational age. Coagulopathy, as defined by INR and platelet count, was not significantly associated with MIPH (Table 2). A higher percentage of patients with MIPH had ETV (18\% vs 1\%, $\mathrm{p}=0.02)$ or a ventriculosubgaleal shunt $(18 \%$ vs $3 \%, \mathrm{p}=0.02)$ compared with patients without MIPH. The distribution of etiology of hydrocephalus differed significantly between the 2 groups $(\mathrm{p}=0.04)$. Aqueductal stenosis was the most frequently observed etiology of hydrocephalus among patients with MIPH (45\% vs $21 \%$ ), whereas CM-II was more frequently observed among those who did not develop MIPH (51\% vs 27\%).

\section{Frontal and Occipital Horn Ratio}

A comparison of FOR measurements in MIPH patients versus controls is shown in Table 3. The preoperative oc- cipital horn measurements were significantly higher in the MIPH group than in the control group $(88.1 \mathrm{~mm}$ vs 67.2 $\mathrm{mm}, \mathrm{p}=0.02$ ). The preoperative FOR was also higher in the MIPH group ( 0.65 vs $0.57, \mathrm{p}<0.001)$. The difference between pre- and postoperative occipital horn width was significantly greater in the MIPH group $(26.2 \mathrm{~mm}$ vs 11.6 $\mathrm{mm}, \mathrm{p}=0.01$ ). As expected, the difference between preand postoperative FOR was also significantly greater in patients with MIPH (0.14 vs $0.08, \mathrm{p}=0.04)$.

The relationship between the etiology of hydrocephalus and FOR measurements is shown in Table 4. There was an overall association between hydrocephalus etiology and preoperative FOR measure $(\mathrm{p}<0.001)$. Patients with aqueductal stenosis had a significantly higher average pre-

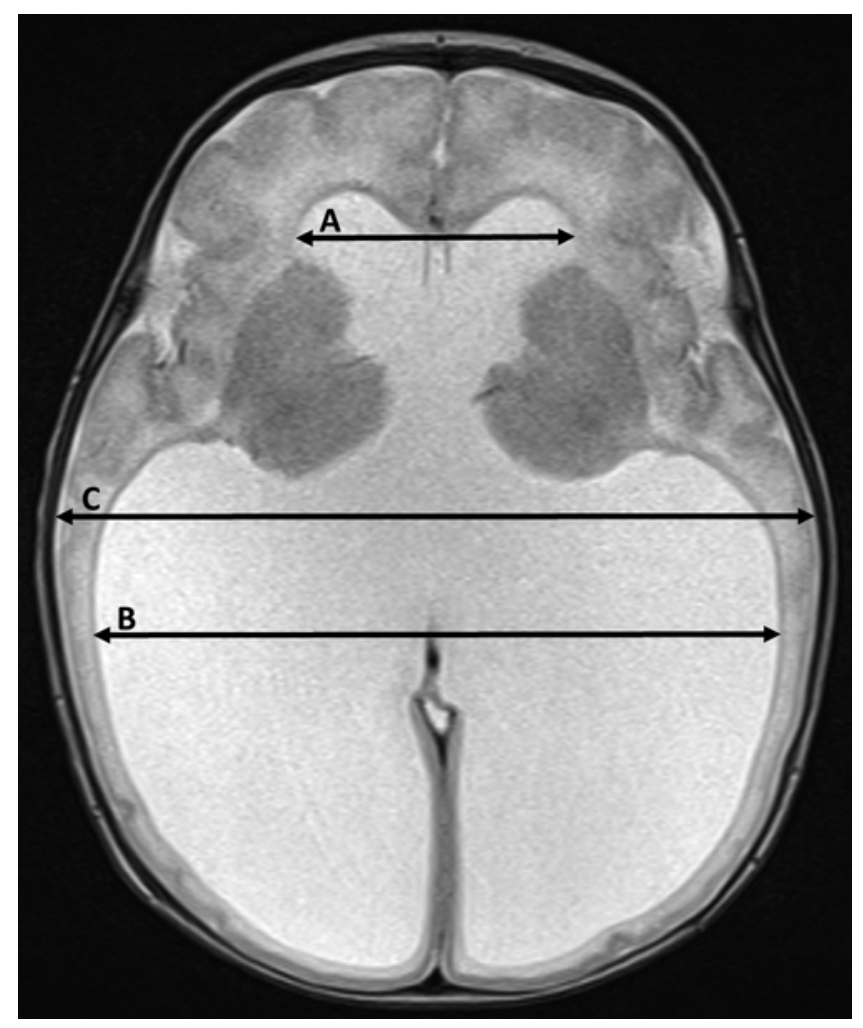

FIG. 2. Axial T2-weighted MR image demonstrating the method used for calculating FOR. The width of the frontal (A) and occipital (B) lateral horns and the biparietal diameter of the brain $(C)$ were measured on preand postoperative images. The sum of the measured width of the frontal (A) and occipital (B) lateral horns was divided by the measured biparietal diameter (C) to yield the FOR. 
TABLE 2. Demographic and clinical characteristics of 121 patients

\begin{tabular}{|c|c|c|c|}
\hline Characteristic & $\begin{array}{l}\text { MIPH } \\
(n=11)\end{array}$ & $\begin{array}{l}\text { No MIPH } \\
(n=110)\end{array}$ & $p$ Value \\
\hline Male sex & $5(45 \%)$ & $54(49 \%)$ & $>0.99$ \\
\hline $\begin{array}{l}\text { Corrected age in wks, } \\
\text { mean (SD) }\end{array}$ & $38.8(7.4)$ & $39.1(3.1)$ & 0.9 \\
\hline Type of CSF diversion & & & 0.02 \\
\hline ETV & $2(18 \%)$ & $1(1 \%)$ & \\
\hline Peritoneal & $7(64 \%)$ & $106(96 \%)$ & \\
\hline Subgaleal & $2(18 \%)$ & $3(3 \%)$ & \\
\hline $\begin{array}{l}\text { Head circumference in cm, } \\
\text { mean (SD) }\end{array}$ & $41.3(6.5)$ & $41.3(6.5)$ & 0.12 \\
\hline Bulging anterior fontanelle & $6(55 \%)$ & $6(55 \%)$ & 0.33 \\
\hline Preterm birth & $3(27 \%)$ & $3(27 \%)$ & $>0.99$ \\
\hline $\begin{array}{l}\text { Gestational age in wks, } \\
\text { mean (SD) }\end{array}$ & $35.3(5.9)$ & $35.3(5.9)$ & 0.41 \\
\hline Etiology of hydrocephalus & & & 0.04 \\
\hline CM-II & $3(27 \%)$ & $56(51 \%)$ & \\
\hline IVH & $1(9 \%)$ & $16(15 \%)$ & \\
\hline Aqueductal stenosis & $5(45 \%)$ & $23(21 \%)$ & \\
\hline $\begin{array}{l}\text { Arachnoid \& intraven- } \\
\text { tricular cysts }\end{array}$ & $0(0 \%)$ & $9(8 \%)$ & \\
\hline Dandy-Walker & $0(0 \%)$ & $3(3 \%)$ & \\
\hline Tumor & $0(0 \%)$ & $2(2 \%)$ & \\
\hline Other & $2(18 \%)$ & $1(1 \%)$ & \\
\hline Shunt valve classification & & & $<0.001$ \\
\hline Low pressure & $1(14 \%)$ & $67(64 \%)$ & \\
\hline Medium pressure & $1(14 \%)$ & $24(23 \%)$ & \\
\hline High pressure & $1(14 \%)$ & $4(4 \%)$ & \\
\hline Flow control & $4(57 \%)$ & $10(10 \%)$ & \\
\hline Coagulopathy at surgery & $1(9 \%)$ & $15(15 \%)$ & $>0.99$ \\
\hline $\begin{array}{l}\text { Preop platelet count, mean } \\
\text { (SD) }\end{array}$ & $339.9(206.7)$ & $363.6(175.5)$ & 0.77 \\
\hline $\begin{array}{l}\text { Preoperative INR, geomet- } \\
\text { ric mean }(95 \% \mathrm{Cl})\end{array}$ & $1.1(1-1.14)$ & $1.1(1-1.14)$ & 0.47 \\
\hline Shunt revision surgery & $1(9 \%)$ & $3(3 \%)$ & 0.32 \\
\hline
\end{tabular}

Values are counts and percentages unless otherwise specified.

operative FOR than patients with CM-II $(\mathrm{p}<0.001)$ and intraventricular hemorrhage $(\mathrm{p}=0.007)$. Hydrocephalus etiology and postoperative FOR were also associated $(\mathrm{p}=$ 0.005). Patients with aqueductal stenosis had significantly higher postoperative FOR measures than patients with CM-II $(p=0.005)$, and $\triangle F O R$ after CSF diversion was not associated with the etiology of hydrocephalus $(\mathrm{p}=0.87)$.

\section{Shunt Valves}

There was a significant association between pre- and postoperative FOR and shunt classification $(\mathrm{p}<0.001$, Table 5). The preoperative FOR in those patients who received medium-pressure valves was significantly higher than that in those patients who received low-pressure valves $(\mathrm{p}=0.003)$. Similarly, the preoperative FOR in those
TABLE 3. Ventricular measures

\begin{tabular}{lrcc}
\hline \multicolumn{1}{c}{ Measure } & $\begin{array}{r}\text { No MIPH } \\
(\mathrm{n}=110)\end{array}$ & $\begin{array}{c}\text { MIPH } \\
(\mathrm{n}=11)\end{array}$ & $\begin{array}{c}\mathrm{p} \\
\text { Value }\end{array}$ \\
\hline Preop frontal horn width & $43.4(13.1)$ & $50.5(14)$ & 0.14 \\
\hline Preop occipital horn width & $67.2(20.6)$ & $88.1(25)$ & 0.02 \\
\hline Preop biparietal diameter & $95.7(13.9)$ & $106.1(21.8)$ & 0.15 \\
\hline Preop FOR & $0.57(0.1)$ & $0.65(0.06)$ & $<0.001$ \\
\hline Postop frontal horn width & $36.7(11.5)$ & $38.0(10.5)$ & 0.71 \\
\hline Postop occipital horn width & $57.1(20.2)$ & $61.9(19.8)$ & 0.47 \\
\hline Postop biparietal diameter & $93.6(12.6)$ & $97.3(15.2)$ & 0.46 \\
\hline Postop FOR & $36.7(11.5)$ & $38.0(10.5)$ & 0.71 \\
\hline Difference in frontal horn width & $7.3(10.4)$ & $12.5(11.6)$ & 0.18 \\
\hline Difference in occipital horn width & $11.6(11.8)$ & $26.2(16.1)$ & 0.01 \\
\hline Difference in biparietal diameter & $2.9(8.3)$ & $8.8(12.3)$ & 0.15 \\
\hline$\Delta$ FOR & $0.08(0.1)$ & $0.14(0.1)$ & 0.04 \\
\hline
\end{tabular}

Values are mean (SD) and measured in millimeters.

patients who received high-pressure valves was greater than in those who received low-pressure valves $(\mathrm{p}<0.001)$. The postoperative FOR measurements in patients with high- and medium-pressure valves were higher than that in those who received low-pressure valves. There was no significant association between $\triangle F O R$ and valve classification $(p=0.26)$. The type of shunt valve was also associated with the presence of MIPH ( $\mathrm{p}<0.001)$. Fifty-seven percent of patients with MIPH had a flow-control valve, while $64 \%$ of patients without MIPH had a low-pressure valve.

\section{Multivariable Models}

Because hydrocephalus etiology, shunt type, shunt classification, and preoperative FOR and $\triangle F O R$ measurements were all associated with MIPH in the univariate case, a multivariable model was fit to assess the risk factors for MIPH. Given the small MIPH sample size $(\mathrm{n}=11)$, the hydrocephalus etiology categories were collapsed into CM-II, aqueductal stenosis, and other. A logistic regression model was fit with MIPH as the outcome, preoperative FOR as the primary explanatory variable, and the following covariates: collapsed hydrocephalus etiology, shunt type (ETV vs peritoneal vs subgaleal), and corrected age

TABLE 4. FOR measurements by etiology of hydrocephalus

\begin{tabular}{|c|c|c|c|}
\hline Etiology & $\begin{array}{c}\text { Preop } \\
\text { FOR }\end{array}$ & $\begin{array}{c}\text { Postop } \\
\text { FOR }\end{array}$ & $\Delta \mathrm{FOR}$ \\
\hline CM-II & $0.5(0.08)$ & $0.5(0.08)$ & $0.09(0.09)$ \\
\hline IVH & $0.5(0.1)$ & $0.5(0.1)$ & $0.08(0.09)$ \\
\hline Aqueductal stenosis & $0.6(0.09)$ & $0.6(0.1)$ & $0.1(0.09)$ \\
\hline Arachnoid \& intraventricular cysts & $0.6(0.1)$ & $0.5(0.1)$ & $0.05(0.07)$ \\
\hline Dandy-Walker & $0.7(0.06)$ & $0.6(0.06)$ & $0.1(0.07)$ \\
\hline Tumor & $0.5(0.2)$ & $0.4(0.1)$ & $0.07(0.01)$ \\
\hline Other & $0.6(0.07)$ & $0.5(0.06)$ & $0.06(0.06)$ \\
\hline$p$ value & $<0.001$ & 0.005 & 0.868 \\
\hline
\end{tabular}

Values are mean (SD). 
TABLE 5. FOR measurements by shunt type

\begin{tabular}{lccccc}
\hline \multicolumn{1}{c}{ FOR } & Low Pressure $(n=77)$ & Medium Pressure $(n=34)$ & High Pressure $(n=14)$ & Flow Control $(n=23)$ & $p$ Value \\
\hline Preop FOR & $0.5(0.09)$ & $0.6(0.08)$ & $0.7(0.07)$ & $0.6(0.1)$ & $<0.001$ \\
\hline Postop FOR & $0.4(0.1)$ & $0.6(0.07)$ & $0.6(0.1)$ & $0.5(0.09)$ & $<0.001$ \\
\hline$\Delta$ FOR & $0.1(0.09)$ & $0.06(0.06)$ & $0.1(0.08)$ & $0.07(0.1)$ & 0.257 \\
\hline
\end{tabular}

Values are mean (SD).

(Table 6). After adjusting for etiology, shunt type, and corrected age, we found that preoperative FOR was not significantly associated with the occurrence of MIPH. Though not statistically significant, there was an increase in the odds of MIPH by 1.7 (95\% CI 0.9-3.1, p = 0.096) for every 1 -unit increase in preoperative FOR. The use of a subgaleal shunt, in comparison with a peritoneal shunt, increased the odds of MIPH by 1.4 (95\% CI 1.1-1.9, p = 0.02) and the use of an ETV, in comparison with a peritoneal shunt, increased the odds of MIPH by 1.7 (95\% CI 1.2-2.5, $\mathrm{p}=$ $0.003)$. The same analysis was performed with $\triangle F O R$, and after adjusting for etiology, shunt type, and corrected age, we found that $\triangle F O R$ was significantly associated with the occurrence of MIPH (Table 7). For every 1-unit increase in $\triangle \mathrm{FOR}$, the odds of MIPH increased by 2.5 (95\% CI $1.2-5.3, \mathrm{p}=0.016$ ).

\section{Discussion \\ Patient Population}

Intraparenchymal bleeding following shunt placement is most commonly observed around the path of the ventricular catheter, and it is thought to be due to catheter advancement through brain parenchyma. ${ }^{5,7-9} \mathrm{MIPH}$, however, represents a hemorrhagic complication of VP shunting with an undefined etiology. Choi et al. ${ }^{2}$ found the incidence of MIPH in 330 pediatric patients under the age of 18 years to be $1.2 \%, 2.9 \%$ for infants below the age of 1 year, and $5.3 \%$ in neonates with a corrected age of less than 3 months. The most common etiology of hydrocephalus for patients in their cohort who developed MIPH was infantile posthemorrhagic hydrocephalus and congenital hydrocephalus without intracranial hemorrhage. The incidence of MIPH in our study population was higher, $9.1 \%$, though we focused on a younger population, neonates (0-30 days of age) who underwent CSF diversion. In our cohort, the most common etiology for hydrocephalus was aqueductal stenosis; the secondmost common etiology was CM-II. In contrast to Choi et al., we found no

TABLE 6. Multivariable logistic regression (outcome: MIPH)

\begin{tabular}{lll}
\hline \multicolumn{1}{c}{ Explanatory Variable } & OR $(95 \% \mathrm{Cl})$ & $\mathrm{p}$ Value \\
\hline Aqueductal stenosis (vs CM-II) & $1(0.9-1.2)$ & 0.5619 \\
\hline All other etiologies (vs CM-II) & $1(0.9-1.1)$ & 0.6813 \\
\hline Subgaleal shunt (vs peritoneal) & $1.4(1.1-1.9)$ & 0.0234 \\
\hline ETV (vs peritoneal shunt) & $1.7(1.2-2.5)$ & 0.0027 \\
\hline Corrected age (days) & $1(1-1)$ & 0.7089 \\
\hline Preop FOR & $1.7(0.9-3.1)$ & 0.0961 \\
\hline
\end{tabular}

significant association between prematurity and incidence of MIPH.

\section{Frontal and Occipital Horn Ratio}

Specific imaging factors, such as severity of ventriculomegaly (FOR) and ventricular response to CSF diversion $(\triangle \mathrm{FOR})$, were found to be significantly associated with MIPH. In the univariate analysis, patients who developed MIPH had a significantly higher preoperative FOR, a marker for the severity of ventriculomegaly. To that end, aqueductal stenosis, the most frequent etiology among MIPH patients, was shown to have a relatively higher preoperative FOR. The $\triangle F O R$ was also significantly higher in patients who developed MIPH. The observed association of $\triangle$ FOR following CSF diversion with MIPH suggests that rapid decompression of the ventricles and subsequent alteration in intracranial pressure and CSF flow dynamics may play a role in the development of these hemorrhages. Interestingly, after multivariable analysis, preoperative FOR did not remain significantly associated with the development of MIPH. Patients with MIPH had higher preoperative FOR and a significantly higher presence of aqueductal stenosis and subgaleal diversions. The multivariable model shows that shunt type is more strongly associated with MIPH than preoperative FOR is. After adjusting for hydrocephalus etiology, shunt type, and patient age, we found that $\triangle F O R$ remained significantly associated with MIPH, suggesting that overly rapid ventricular decompression is related to its development.

\section{CSF Diversion and Shunt Valves}

To minimize rapid decompression and associated sequelae, neurosurgeons employ various strategies to prevent overdrainage of CSF during shunt implementation and in the early postoperative period. These strategies include, but are not limited to, minimizing intraoperative CSF loss, employing adjustable ("programmable") valves set to high initial opening pressures, and keeping patients horizontal
TABLE 7. Multivariable logistic regression (outcome: MIPH)

\begin{tabular}{lll}
\hline \multicolumn{1}{c}{ Explanatory Variable } & OR $(95 \% \mathrm{Cl})$ & $\mathrm{p} \mathrm{Value}$ \\
\hline Aqueductal stenosis (vs CM-II) & $1.1(0.9-1.3)$ & 0.2072 \\
\hline All other etiologies (vs CM-II) & $1(0.9-1.2)$ & 0.9184 \\
\hline Subgaleal shunt (vs peritoneal) & $1.3(1-1.8)$ & 0.1021 \\
\hline ETV (vs peritoneal shunt) & $1.9(1.3-2.7)$ & 0.0017 \\
\hline Corrected age (days) & $1(1-1)$ & 0.9876 \\
\hline$\Delta$ FOR & $2.5(1.2-5.3)$ & 0.0164 \\
\hline
\end{tabular}


for various lengths of time after implantation to minimize siphon effects. In our multivariable analysis, we compared 3 methods of CSF diversion (subgaleal shunts, VP shunts, and ETV) and their association with MIPH. Interestingly, more patients who developed MIPH were treated with CSF diversion techniques lacking a valve providing resistance to CSF flow-ETV and subgaleal shunts. More than half (57\%) of the patients with MIPH had flow-control valves, while the majority of those without MIPH had low- or medium-pressure valves. Regarding VP shunt valves, highpressure valves and flow-control valves were used more frequently in MIPH patients, whereas low- and mediumpressure shunt systems were more common in patients without MIPH. Choi et al. ${ }^{2}$ recommend setting programmable valves to the highest pressure levels in the immediate postoperative period, gradually decreasing to the desired pressure level. We must exercise caution in interpretation of the data presented here, as they are potentially confounded by neurosurgeons' selection bias-for example, the use of higher-pressure shunt valves in high-risk patients, including those with higher FOR observed on preoperative imaging. The association of high-pressure valves with MIPH may indicate surgeons' attempts to use high-pressure resistance to temper the rate of ventricular decompression, but it also suggests that these efforts are insufficient to prevent hemorrhages. Additionally, the amount of time that patients were kept flat and immobile following CSF diversion could not be determined in the present study and may be a significant contributing risk factor.

\section{MIPH Pathophysiology}

MIPH is observed apart from neonatal shunted hydrocephalus; a similar phenomenon is described in the adult literature in patients who have undergone decompression of chronic subdural hematomas. ${ }^{6,11}$ Several hypotheses have been postulated as to the etiology of the parenchymal hemorrhages: 1) perfusion breakthrough bleeding, 2) the effects of disrupted cerebral vascular autoregulation, 3) vascular damage secondary to the mechanical shift of cranial contents, and 4) the interplay between intracranial and venous pressures. ${ }^{2-4}$

Choi et al. ${ }^{2}$ hypothesized that MIPH in infants is likely related to increased cerebral perfusion pressure and a premature brain vasculature with limited tolerance to alterations in perfusion. ${ }^{2}$ Based on the subcortical and periventricular location of the MIPH, we suggest that the bleeds are venous in etiology. We postulate that with increased parenchymal blood flow and altered pressure dynamics following CSF diversion, neonatal intracerebral veins are vulnerable to rupture; this venous vulnerability is a reflection of disrupted autoregulation and leads to a type of perfusion breakthrough bleeding. A plausible explanation is that venous stretch secondary to mechanical decompression in the setting of variable ventricular compliance may contribute to postoperative parenchymal bleeding, but direct evidence of this mechanism has yet to be demonstrated in humans or animal models of hydrocephalus.

\section{Clinical Follow-Up}

The long-term clinical outcome of patients with MIPH is largely unknown. Choi et al. ${ }^{2}$ discussed potential long- term negative effects of MIPH, but no long-term follow-up was completed to determine functional status. Our data suggest that MIPH may be associated with a higher rate of shunt revision within a year of initial CSF diversion (Table 2); however, we were limited by the quantity of follow-up data. As hydrocephalus is a heterogeneous entity, further study of patient-specific and treatment-related parameters in larger patient populations, including those with elevated FOR and aqueductal stenosis, is needed to develop preventative strategies.

\section{Study Method Limitations}

The findings from this study are subject to typical limitations of retrospective studies and the small number of MIPH patients. Despite the many statistical tests performed, $\mathrm{p}$ values were not adjusted, due to the exploratory nature of the study. In addition, no a priori power analysis was performed, so the study may not have adequate power to detect significant findings. Further, the surgeons' selection bias likely acts as confounding factor. For these reasons, a prospective study of MIPH prevention strategies with an assessment of possible implications for patient outcomes is needed. There is also a selection bias as to which neonates underwent imaging studies and at what time point after shunt placement this occurred, which is inherent to our study.

\section{Conclusions}

MIPH represents a not-infrequent complication of neonatal shunted hydrocephalus. Regardless of hydrocephalus etiology, shunt type, and patient age, $\triangle F O R$ after shunt placement, but not preoperative FOR, was associated with MIPH in this study. An association between highpressure and flow-control valves was found with MIPH among patients with neonatal hydrocephalus, likely representing surgeon selection bias for patients with severe ventriculomegaly. Inherent to this suspected bias, our findings advocate for further study of modulation of $\triangle F O R$ after shunting, either via magnitude or rate of ventricular size change, to determine significant modifiable risk factors in mitigating the risk of MIPH. A recent retrospective analysis demonstrated that among children under 18 years of age the percentage of $\triangle F O R$ is correlated with a need for early shunt revision (within 90 days of placement), although the etiology of this proclivity for early shunt failure is unknown. ${ }^{13}$ Changing the magnitude and rate of ventricular size change will require an intelligent paradigm for shunt valve selection. Measurement of ventricular opening pressure and compliance may prove useful in this regard, but the association between intracranial pressure and ventricular size and measurement indices is incompletely understood. ${ }^{14}$ A prospective study of potential MIPH prevention strategies and assessment of possible implications for long-term patient outcome is needed.

\section{Acknowledgments}

We wish to acknowledge Jacqueline A. Bello, MD, for her inspiration and mentorship. 


\section{References}

1. Borgbjerg BM, Gjerris F, Albeck MJ, Hauerberg J, Børgesen SE: Frequency and causes of shunt revisions in different cerebrospinal fluid shunt types. Acta Neurochir (Wien) 136:189-194, 1995

2. Choi JW, Kim SK, Wang KC, Lee JY, Cheon JE, Phi JH: Multifocal intraparenchymal hemorrhages after ventriculoperitoneal shunt surgery in infants. J Neurosurg Pediatr 14:329-335, 2014

3. Cook AW, Browder EJ, Carter WB: Cerebral swelling and ventricular alterations following evacuation of intracranial extracerebral hematoma. J Neurosurg 19:419-423, 1962

4. d'Avella D, De Blasi F, Rotilio A, Pensabene V, Pandolfo $\mathrm{N}$ : Intracerebral hematoma following evacuation of chronic subdural hematomas. Report of two cases. J Neurosurg 65:710-712, 1986

5. Fukamachi A, Koizumi H, Nukui H: Postoperative intracerebral hemorrhages: a survey of computed tomographic findings after 1074 intracranial operations. Surg Neurol 23:575-580, 1985

6. Kim JK, Kim SW, Kim SH: Intracerebral hemorrhage following evacuation of a chronic subdural hematoma. J Korean Neurosurg Soc 53:108-111, 2013

7. Kubokura T, Nishimura T, Koyama S, Sanno N, Tsubone K: [Delayed intracerebral hemorrhage following VP shunt operation.] No Shinkei Geka 16 (5 Suppl):523-527, 1988 (Jpn)

8. Lund-Johansen M, Svendsen F, Wester K: Shunt failures and complications in adults as related to shunt type, diagnosis, and the experience of the surgeon. Neurosurgery 35:839844, 1994

9. Misaki K, Uchiyama N, Hayashi Y, Hamada J: Intracerebral hemorrhage secondary to ventriculoperitoneal shunt insertion-four case reports. Neurol Med Chir (Tokyo) 50:7679, 2010

10. O'Hayon BB, Drake JM, Ossip MG, Tuli S, Clarke M: Frontal and occipital horn ratio: A linear estimate of ventricular size for multiple imaging modalities in pediatric hydrocephalus. Pediatr Neurosurg 29:245-249, 1998

11. Ogasawara K, Koshu K, Yoshimoto T, Ogawa A: Transient hyperemia immediately after rapid decompression of chronic subdural hematoma. Neurosurgery 45:484-489, 1999

12. Okuyama T, Hashi K, Sasaki S, Sudo K, Kurokawa Y: Changes in cerebral microvasculature in congenital hydrocephalus of the inbred rat LEW/Jms: light and electron microscopic examination. Surg Neurol 27:338-342, 1987

13. Patra DP, Bir SC, Maiti TK, Kalakoti P, Cuellar H, Guthikonda $\mathrm{B}$, et al: Role of radiological parameters in predicting overall shunt outcome after ventriculoperitoneal shunt insertion in pediatric patients with obstructive hydrocephalus. Neurosurg Focus 41(5):E4, 2016
14. Sæhle T, Eide PK: Characteristics of intracranial pressure (ICP) waves and ICP in children with treatment-responsive hydrocephalus. Acta Neurochir (Wien) 157:1003-1014, 2015

15. Tuli S, Drake J, Lawless J, Wigg M, Lamberti-Pasculli M: Risk factors for repeated cerebrospinal shunt failures in pediatric patients with hydrocephalus. J Neurosurg 92:31-38, 2000

16. Udvarhelyi GB, Wood JH, James AE Jr, Bartelt D: Results and complications in 55 shunted patients with normal pressure hydrocephalus. Surg Neurol 3:271-275, 1975

17. Wong JM, Ziewacz JE, Ho AL, Panchmatia JR, Bader AM, Garton HJ, et al: Patterns in neurosurgical adverse events: cerebrospinal fluid shunt surgery. Neurosurg Focus 33(5):E13, 2012

18. Wozniak M, McLone DG, Raimondi AJ: Micro- and macrovascular changes as the direct cause of parenchymal destruction in congenital murine hydrocephalus. J Neurosurg 43:535-545, 1975

\section{Disclosures}

The authors report no conflict of interest concerning the materials or methods used in this study or the findings specified in this paper.

\section{Author Contributions}

Conception and design: Mirsky, Parker. Acquisition of data: Mirsky, Oushy, Stence. Analysis and interpretation of data: Mirsky, Oushy, Parker, Campbell, Palmer, Stence. Drafting the article: Oushy. Critically revising the article: Oushy, Parker, Wilkinson, Handler. Reviewed submitted version of manuscript: Mirsky, Palmer, Wilkinson, Stence, Handler. Statistical analysis: Campbell, Palmer. Administrative/technical/material support: Oushy, Wilkinson. Study supervision: Mirsky, Oushy.

\section{Supplemental Information}

\section{Previous Presentations}

Portions of this work were presented in abstract form at the 2016 Annual Meeting of the Congress of Neurological Surgeons, San Diego, California, September 24-28.

\section{Correspondence}

David M. Mirsky, Children's Hospital Colorado, 13123 East 16th Ave., Box B125, Aurora, CO 80045. email: david.mirsky@ childrenscolorado.org. 